\title{
ASSESSMENT OF KNOWLEDGE, PRACTICES, AND ATTITUDE OF HAND HYGIENE AMONG UNIVERSITY NURSING AND OPTOMETRY UNDERGRADUATE STUDENTS IN THEIR FINAL YEAR OF TRAINING
}

\author{
Janitha Plackal Ayyappan ${ }^{1}$, Virgina Varghese ${ }^{2}$ and Galal Mohammed Ismail ${ }^{3}$ \\ ${ }^{1}$ Department of Optometry, College Of Health Sciences, University of Buraimi, Al Buraimi, Sultanate of \\ Oman. \\ ${ }^{2}$ Department of Nursing, College Of Health Sciences, University of Buraimi, Al Buraimi, Sultanate of \\ Oman. \\ ${ }^{3}$ College Of Health Sciences, University of Buraimi, Al Buraimi, Sultanate of Oman.
}

Corresponding author: Janitha Plackal Ayyappan

Email: ravijani@gmail.com; virgina.varghese@gmail.com

\begin{abstract}
Safe Hand hygiene practice is considered the most essential component of patient safety. Especially when it comes to the health professionals; students are exposed to their clinical practice in the first years of their education. This accelerates the chances of getting infection via cross transmission of hands through the healthcare worker to the patient is most common. A quantitative cross sectional, questionnaire-based survey was carried out to investigate the level of Knowledge, practices, and attitude of Hand hygiene among university nursing and optometry undergraduate students in their final year of training. A total of one hundred and forty three subjects took part in the survey of which 73 nursing students and 70 optometry students. Fisher's exact test was applied to test the level of Knowledge on practice and attitude. And the correlation coefficient $r=-1$ to +1 with $P>0.05$ was obtained. To add, using an observational checklist will be the ideal way to assess the hand hygiene practice in the clinical area. Moreover add a mandatory component as patient safety into the curricula of the undergraduate students of allied health will enhance the patient safety more effectively.
\end{abstract}

Keywords: Knowledge, attitude, practices, Hand hygiene, Nursing and Optometry, undergraduate students

\section{INTRODUCTION}

Globally millions of populace jeopardy due to the negligence of simple hand hygiene exercise not appropriately followed in a routine manner. Because of following improper hand wash technique high infection rate in a developed and developing world; it's varying between 10 to 50 percent respectively [1]. The conventional way of maintaining proper hand hygiene among healthcare people to keep a safe and neat hand by using a soap and water or using alcohol-based solutions. This fact is supported by several researchers and the literature; conversely; following this method can minimise the transmission of the pathogen, which spread through the health professional. Moreover; these norms are not succeeding due to poor acquiescence [2].

To overcome these challenges; adequate training should be given in the health sectors mainly people who work or handle in the areas of hand hygiene practice for the students who practice or learners of hand wash as an initial stage to assure the high-level efficacy. There are mainly three hand hygiene practice followed worldwide such as, hand wash with soap and water, with antiseptic solution and surgical handwashing [3]. However; in this study is focused on normal hand hygiene technique with soap and water, where the handwashing will be performed for 20 to 30 seconds using eight steps according to the recommendations of WHO which is proven effective against microorganism [4]. Interestingly; numerous studies have been conducted to find the Knowledge, attitude and practices of hand hygiene among various health science students to comprehend the underlying cause of infections which spread through the hands of a student's mainly via crosstransmission. According to CDSC Oman high priority was given to patient safety program especially hand hygiene in the country. In order to promote hand hygiene various community level events were conducted across the country to create awareness and educate the people about the importance of hand hygiene in Oman [5].

As a part of effective and best academic practices, across GCC (Gulf cooperation council) highest priority was given to patient safety. When it comes to Oman context, all higher educational institutions are offering health care Programmes; as mandatory patient safety plugged into existing curricula as an integral part of teachingThe curricula adapted from WHO patient safety curriculum guidelines [3]. More importantly, very few studies have been conducted, to assess the knowledge, attitude and practices between undergraduate students at university level in Oman. Moreover; students during the final year of 
training in the health sectors are undergone rigorous ongoing and hands-on practices sessions, to gain adequate experience in their field of expertise. During this transit period, seldom may fail to recall the hand hygiene during the patient care. Optometry and Nursing were the two undergraduate program available in the study settings. Furthermore, from the health science, student population researcher wanted to know the readiness of the health professional going to practice.

The purpose of the study is to assess evaluate Knowledge, practices, and attitude of Hand hygiene among university nursing and optometry undergraduate students in their final year of training.

\section{METHODS}

\section{Study Design}

A quantitative, cross-sectional questionnairebased research was carried out to investigate the Knowledge, attitude and practices about hand hygiene among nursing and optometry undergraduate students in their final year of training for one semester (3 months).

Before data collection, Ethical approval for conducting a survey was obtained from College Research and Ethics Committee. Once the formal approval was acquired, the purpose of the study narrated to the participants before administering the questionnaire. Followed by a selfadministered questionnaire containing knowledge, attitude and hand hygiene practices was delivered to the study participants. The survey conducted during the break time of the college, considering not having any impact on the students study hours. Also clarified by the researcher that, while participating in the research for the students not going to have any adverse effect on their academic performances. Also ensures that the researcher does not directly or indirectly involve the educational evaluation of this particular study subjects. By receiving a signed, completed questionnaire along with consent confidentiality was assured to the participants. And this filled survey form was collected in a sealed envelope. A validated selfadministered questionnaire comprises the sociodemographic signatures like the name of the participants, age sex, ID No and program procured from the subjects. However, to take part, the study was purely at the will of participants. In addition to this; the patient information sheet also elucidates that the study subjects have all the rights to discontinue from the study any given time throughout the research duration. Likewise; the principal investigator assures the participants at the time of administering the questionnaire that, this particular data will be only used for academic or research just and not for commercial aspects. And the hard copies of the patient information details like candidate signed consent form containing the name preserved in a secure and inaccessible place from the other research data. A pilot study conducted for 20 sample; 10 from each group was considered (Optometry and Nursing) to check the reliability. And to check the internal consistency Cron Bach's Alpha was applied (Attached in the Appendices) and it found the test was significant 0.870 .

\section{Data Collection}

The questionnaire used in the study had a total of four main components; section-I containing Sociodemographic details and the section II consisted of a knowledge questionnaire. The current research knowledge part was evaluated using a WHO hand hygiene questionnaire for healthcare workers. The knowledge section questionnaire consists of a total of 25 items. Out of these; three items are having only responses and fourteen items having YES or NO reactions, four items having a TRUE or FALSE answer. The remaining four questions got only correct answers. The items in which only responses like RUBBING and NONE were given the score value one. Similarly, the YES or NO replies also given the score value of the entire correct responses of YES or NO $=1$ and the incorrect answers of $\mathrm{YES} / \mathrm{NO}=2$. Conversely; the items in which single correct responses gave the score value of correct $=1$ and incorrect $=2$. However; the questions in which YES/ NO answers, TRUE/FALSE replies and single entries like RUBBING and NONE answers only correct statements considered for analysis. While attitude and practices were analysed by a selfdesigned questionnaire; having eight items in each. Similarly, the section-III questions, as well as section-IV questionnaire, was self-designed with the help from a review of the literature and previously published studies. The attitude level as well as the practice level of hand hygiene questionnaire Likert scale scoring used, and the participants were given a choice to select from 0 3. A total of 8 questions having four items like ALWAYS=3, MOST OF THE TIME=2, SOMETIMES=1 AND NEVER $=0$ score given. The participants were given a choice to select from 0 to 3 points. The validity and reliability were estimated, content validation of the questionnaire done by the subject expert and reliability, and the internal consistency was checked by conducting a pilot study and using a Cronbach's Alpha test, and it revealed the significance of 0.870 .

\section{Statistical analysis}

Descriptive statistics were applied in the present research to brief the data. The research subjects narrated by using a mean, standard deviation (SD), proportion, and frequencies with 95\% confidence level $(\mathrm{Cl})$. The primary data includes both categorical and continuous variables. A continuous variable like age was used in the research. The categorical variables like Knowledge, attitude practices, place of the study and gender used in the study. Mean score, standard deviation (SD) estimated for total 
Knowledge, attitude and practices of hand hygiene among nursing vs optometry students. Fisher's exact test was applied to find the level of Knowledge of hand hygiene practices, attitude over hand hygiene practice and practices over hand wash. To find the association of Knowledge over hand hygiene practices and attitude Pearson correlation test performed, and it reveals that $P>0.05$ there is zero correlation between knowledge score with attitude score as well as practice score on hand hygiene.

\section{RESULTS}

Demographic representation of hand hygiene practices

A total of 143 samples were collected for analysis. Out of that; $73(51.04 \%)$ nursing sample and 70

Table 1. Distribution of demographic signature of the sample used in the study
(48.96\%) optometry sample drawn from the College of Health Sciences. Further details are shown in the table-1.

\section{General KAP of Hand hygiene}

The Mean knowledge score was observed in nursing 73 with (11.70) SD \pm 1.769. Optometry knowledge attained 70 (11.09) SD \pm 1.947 . Attitude part Mean score in nursing 73 (19.90) SD \pm 3.267, optometry score 70 (18.07) SD \pm 3.637 . Mean value gained during the total practice score for nursing 73 (6.03) SD \pm 2.115 with optometry 70 (6.56) $\mathrm{SD} \pm 2.811$. Further details are shown in the table-2.

Optometry Nursing Total

$\begin{array}{lll}70(48.96 \%) & 73(51.04 \%) & 143(100 \%)\end{array}$

Table 2. Overall score distribution of knowledge, attitude and practice of hand hygiene among study participants

\begin{tabular}{llrrr}
\hline Overall Score & & N & \multicolumn{1}{c}{ Mean } & \multicolumn{1}{c}{ SD } \\
\hline Total Knowledge Score & Nursing & 73 & 11.70 & 1.769 \\
& Optometry & 70 & 11.09 & 1.947 \\
Total Attitude Score & Nursing & 73 & 19.90 & 3.267 \\
& Optometry & 70 & 18.07 & 3.637 \\
Total Practice Score & Nursing & 73 & 6.03 & 2.115 \\
& Optometry & 70 & 6.56 & 2.811 \\
\hline
\end{tabular}

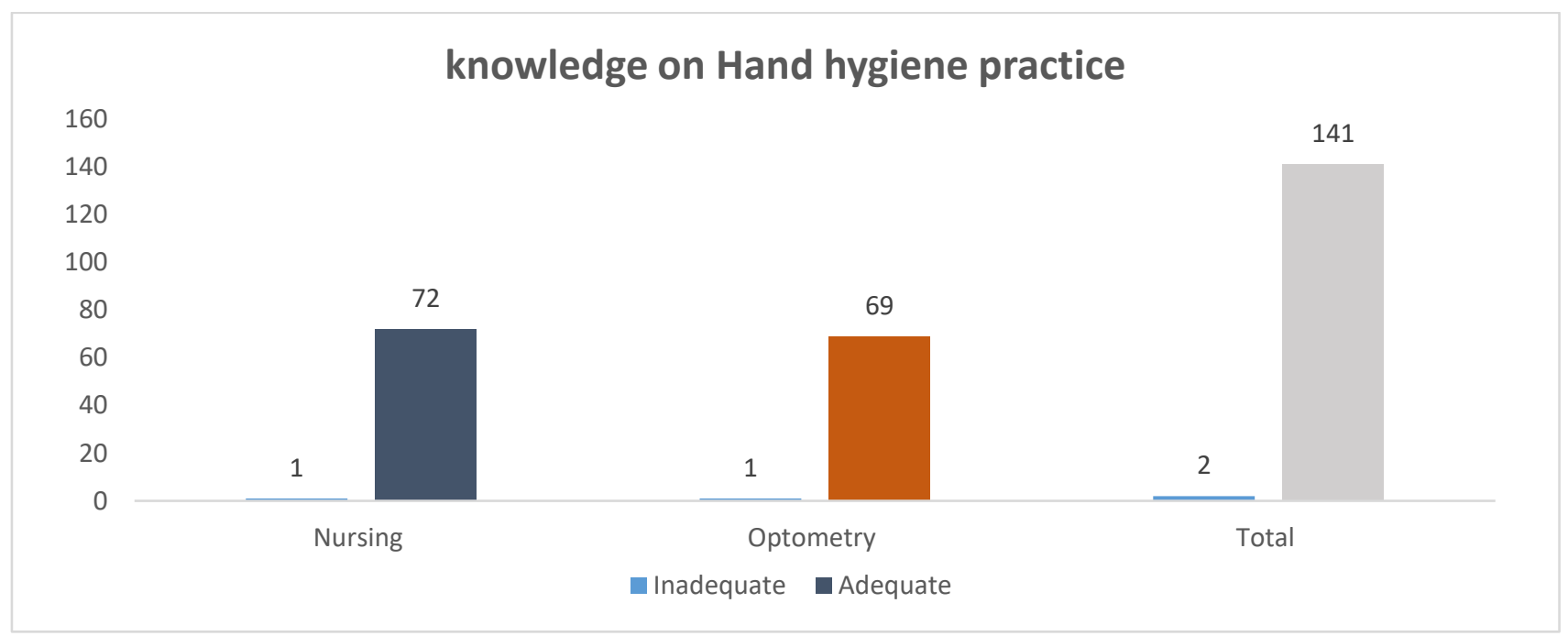

Figure-1 Comparison of knowledge score between nursing vs optometry on hand hygiene practices 
Figure-1 describes the Comparison of knowledge score on hand hygiene practice among optometry and nursing students during their final year of training. Out of 141 samples obtained from optometry; 69 (98.57\%) participants responded adequately to having Knowledge of hand hygiene practices among optometry. While; 72 (98.63\%) from Nursing replied as adequate Knowledge about hand hygiene practices. And $1(1.42 \%)$ study subjects from both the discipline were expressed as poor Knowledge. Moreover; no excellent response was reported from both discipline (optometry as well as Nursing) about the level of Knowledge of hand hygiene practices. To confirm the significance level from both the group about the Knowledge having less than five score item analysis Fisher's exact test was applied and the $p$-value is 0.999 , which showed no significant difference between both the target groups at the $5 \%$ level.

\section{Attitude on Hand Hygiene practice}

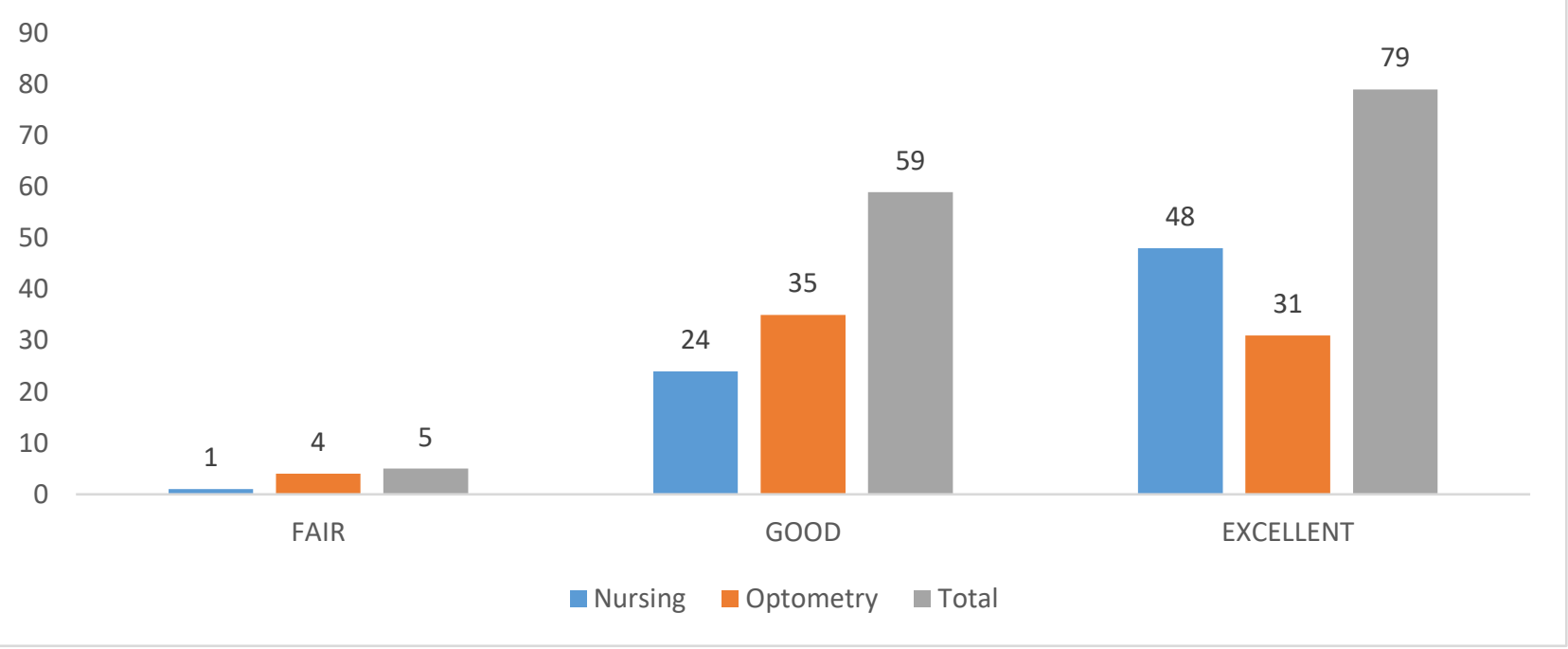

Figure-2 Comparison of attitude score between Nursing Vs Optometry on hand hygiene practices

Figure-2 describes the relationship of attitude score on hand hygiene practice among optometry and nursing students during their final year of training. Over $79(55.24 \%)$ responses received from nursing $48(65.75 \%)$ found an EXCELLENT attitude about optometry; $31(44.29 \%)$. Whereas; $24(32.88 \%)$ from nursing showed as a GOOD attitude in par with optometry $35(50 \%)$ over $59(41.26 \%)$. When it comes to; FAIR responses; only $1(1.37 \%)$ individual was given compare with optometry $4(5.71 \%)$ having a total of $5(3.5 \%)$. Remarkably; hardly any POOR answer got from both disciplines about attitude measurement on hand hygiene practices. To verify, is there significant variances between attitudes over hand hygiene practices between two clusters having less than five score item analysis Fisher's exact test was carried out having a P-value 0.019, which indicates there is a significant difference in hand practices over attitude between both the group at the level of $5 \%$.

Figure- 3 refers to the association of practice score on hand hygiene among optometry and nursing students during their final year of training. Total $78(54.54 \%)$ subjects drawn from nursing $43(58.9 \%)$ replied as a POOR attitude about optometry; $35(50 \%)$. Whereas $30(41.10 \%)$ from nursing presented as FAIR attitude equates with optometry 34 (48.57\%) over 64(44.76\%). When it comes to GOOD responses, only $1(1.43 \%)$ subjects agreed from optometry while nursing found to be no response. Hardly any excellent reply was received from both discipline about

practice measurement on hand hygiene. To prove, is there any significant deviations between practice over hand hygiene amongst two groups having less than five score item analysis Fisher's exact test performed and the p-value 0.0354 , reveals that no significant difference in hand hygiene over the pattern of practices between both the group at the level of $5 \%$.

Figure-4 demonstrates the connection between Knowledge vs training on hand hygiene. The relationship between Knowledge overtraining on hand hygiene found no significant with $\mathrm{P}$-value 0.999 at the $5 \%$ level. To add, current study finds there is no effect on Knowledge over the practice when it comes to hand hygiene among health science students.

Figure-5 exhibits the relationship between Knowledge vs attitude on hand hygiene. The association between Knowledge over attitude on hand hygiene found no significant with $\mathrm{p}$-value 
0.999 at the $5 \%$ level. This promises in the present study that there is no influence on Knowledge over attitude when it comes to hand hygiene among health science professionals.

To test the correlation between Knowledge over attitude and Practice on hand hygiene Pearson correlation was applied. And the correlation coefficient $r=-1$ to +1 with $P>0.05$. Hence to summarize, there is no correlation statistically observed between Knowledge over attitude and practices.

\section{Practice on Hand Hygiene}

90

80

78

70

60

50

43

40

30

20

10

0

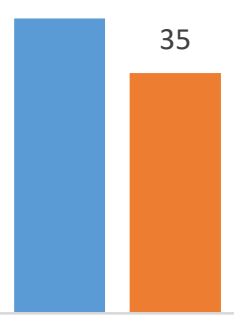

POOR

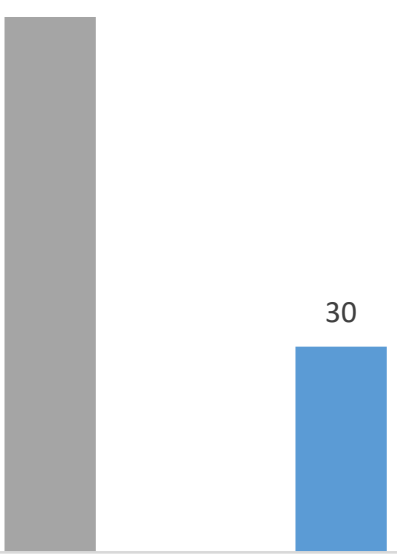

nursing

FAIR

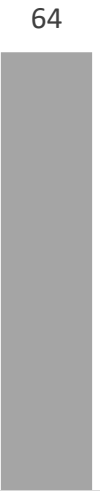

0

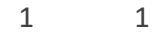

GOOD

1

Figure-3 Recites the Comparison of practice score on hand hygiene between nursing vs optometry students

\section{Association between knowledge vs Practice on Hand hygiene}
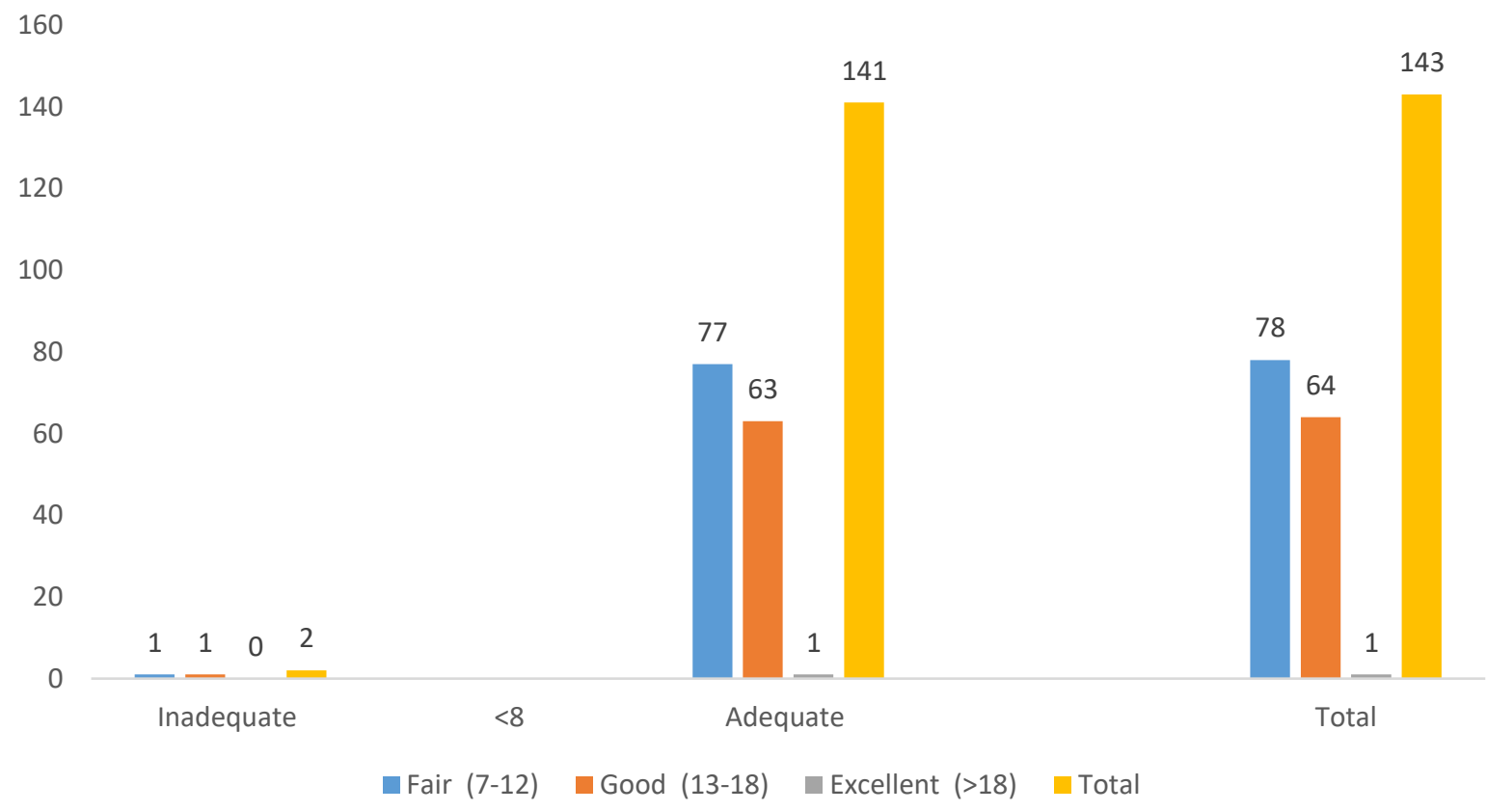

Figure-4 Association between knowledge vs Practice on hand hygiene 


\section{Association between knowledge vs attitude on Hand hygiene}

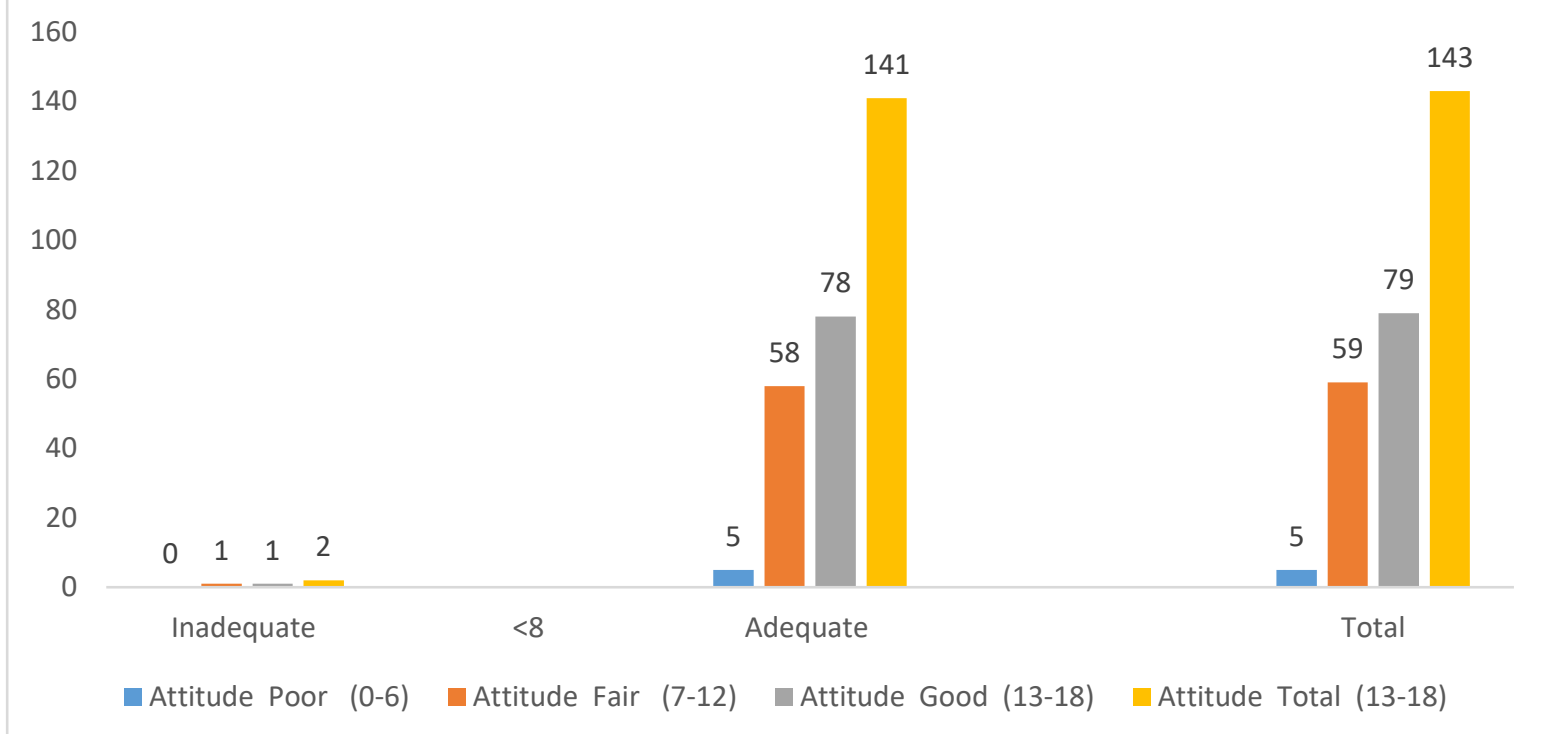

Figure-5 Association between knowledge vs. Attitude on hand hygiene

\section{DISCUSSION}

The current study is to find the level of Knowledge, attitude and practices on hand hygiene practices among university nursing and optometry students in their final year of training. There are several studies reported across the globe about hand hygiene practices among nurses over other medical professional [6]. However; this is the first study conducted in the eastern Mediterranean region (EMRO) to compare the hand hygiene practices among Optometry and nursing students to the best of our Knowledge and from the recent Medline search.

Total 143 responses collected. The present study reveals 141 students articulated an adequate (816) level of Knowledge of hand hygiene practices. Only two subjects responded with inadequate $(<8)$ knowledge on hand hygiene. The knowledge level between both groups, i.e., nursing and optometry found more or less same; 72 and 69 respectively. The minimal difference might be due to both groups were belongs to health science, and moreover, they are in the final year of the clinical training. During the clinical year, these students gained more practical Knowledge and a hands-on session about hand hygiene practice. A study conducted by $[7,8]$ among residents and nursing staff reported that only average Knowledge observed between residents and nurses. Also argues that there is no commendable difference in the level of Knowledge on hand hygiene achieved the Knowledge. The present study showed that there is a significant difference in the level of attitude on hand hygiene practices.

A Total of 79 subjects gained the excellent $(>18)$ score on attitude level on hand hygiene over 143. While 59 participants achieved GOOD (13-18) score on attitude. Five students gained FAIR (7-
12) responses about attitude on hand hygiene practices. The most interesting fact that; no POOR responses obtained from both the group; Nursing and Optometry. While the study done by [6] reported that attitude of a health care professional about hand hygiene needs to strengthen so that serious adverse events due to dirty or unhygienic hand wash practices avoided. Also, the author recommends that vigorous preclinical training included as a part of health education.

The current research emphasises practices over hand hygiene found no significant difference between nursing and optometry with $\mathrm{p}$-value 0.354 at the level of $5 \% .78$ participants responded as poor (0-6) score on hand hygiene practices over 143 . And 64 subjects given the score as fair (7-12) and only one optometry participant was given the score on hand hygiene practices as good (3-18). And no one from both the group gave a score of Excellent $(>18)$. The present study showed that $50 \%$ of the subjects reveal that they received formal training about hand hygiene. Though; the level of Knowledge was found as adequate $98.6 \%$. Similarly, $50 \%$ subjects answered that not received any formal training. Majority of the students who not undergone the training belongs to Optometry discipline.

Another study mentioned about the level of Knowledge in medicos and nursing professional on hand hygiene showed that $80 \%$ with moderate level [9]. Also; nursing professional showed higher Knowledge compared to medicos and this highly correlating with the present study as well. Several studies discuss that hand hygiene knowledge have a huge impact on practices and it is not optimum in our study as well and this is supported by equally the research done by various places like 
Saudi Arabia, Namibia, Brazil, India, China and UK[10]. The current research exhibits $48 \%$ of the health science students uses alcohol-based hand rub to clean the hand regularly. Out of that $26 \%$ of the students only aware the fact that, 20 seconds required to kill the germs in the hand while using an alcohol-based hand rub.

A study on hand hygiene talk about 58.2\%, medical students prefer to use alcohol-based hand rub for hand washing, and only $38.1 \%$ knows that alcoholbased hand rub can eliminate the microbes present in the hands[11]. Present study emphasis that as per WHO, 87\% of the health science students knows that hand hygiene action prevents the transmission of microbes entry if the hand wash performed before touching to the patient [12] Conversely; another researcher mentioned that $53.3 \%$ of medical students aware that following a good hand hygiene practice is essential to inhibit the diseases in the healthcare institutions [13]. The current research assesses the level of Knowledge, attitude and practices of hand hygiene among nursing and Optometry undergraduate students during their final year of training reflects the equal level of Knowledge on hand hygiene practices between both groups and only attitudes found too high among nursing professional.

The key strength of the study; first study to assess the Knowledge, attitude and practices on hand hygiene among undergraduate students at university level in Oman. The sample size calculated for the survey was the only final year of training students. And also for the duration of the survey was taken just one semester; which was very short. The Furthermore, practice should have been assessed using an observational checklist in the clinical setting. Due to paucity of time and personnel, a self-report on practice was used in lieu. Same study could be conducted using a larger and heterogeneous group. The workshops, seminars and hands-on training must be conducted in every academic semester as well as it should be included as a part of the preclinical training as well.

\section{CONCLUSION}

By conducting a study on evaluating the level of Knowledge, attitude and practices about hand hygiene practices among nursing and optometry students during their final year of training, it was revealed that there is no statistically significant difference in Knowledge and practice between nursing and optometry students. However, a statistically significant difference in attitude towards hand hygiene was found between the two groups with nursing scoring higher in the attitude scale. The main motive for selecting optometry and Nursing final year student's populace is the fact that, students who enrolled in the final year of the Programme will be more familiar to the clinical practices mainly the hand hygiene practices and related procedures compared to others. On the other hand, it is vital to test the level of hand hygiene practice for this group, as they are the one who tomorrow's professionals. Furthermore; creating awareness among the health science students about hand hygiene is very important to lessen the hazards of getting infections through the hands of health professionals.

\section{Conflict of interest}

The authors declare no potential conflict of interest.

\section{Acknowledgements}

We thank academic and administrative staff of college of health sciences, participants who voluntarily took part in the study for the accomplishment of research.

\section{Author contributions \\ Janitha plackal ayyappan: conceptualization, methodology, writing, reviewing and editing. \\ Virgina varghese: Data curation, writing an original draft preparation \\ Galal ismail: supervision}

\section{REFERENCES}

1. WHO | Countries or areas running hand hygiene campaigns [Internet]. Who.int. 2020 [cited 14 September 2020]. Available from: https://www.who.int/gpsc/national_campaig ns/country_list/en/.

2. KuKanich KS, Kaur R, Freeman LC, Powell DA. Evaluation of a hand hygiene campaign in outpatient health care clinics. AJN The American Journal of Nursing. 2013 Mar 1;113(3):36-42.

3. Emed.ie. 2021 [cited 10 June 2021]. Available from: https://emed.ie/_docs/ID MUH_Infection_Control_Manual_Jun2006.pdf

4. Ung CO. Community pharmacist in public health emergencies: Quick to action against the coronavirus 2019-nCoV outbreak. Research in Social and Administrative Pharmacy. 2020 Apr 1;16(4):583-6.

5. CDSC OMAN | Infection Control. [online] Available at: http: / /www.cdscoman.org/infectioncontrol.html [Accessed 28 Jul. 2017].

6. Sallami ZA. Assessment of Hand Hygiene Attitude, Knowledge and Practice among Health Science Students in Aden University. Journal of Biosciences and Medicines. 2016 Sep 6;4(9):25-32.

7. WHO EMRO | WHO multi-professional patient safety curriculum guide in the Region, 11-12 March 2012 | oman-news | 
Oman [Internet]. Emro.who.int. 2020 [cited 16 September 2020]. Available from:

http://www.emro.who.int/omn/omannews/patient-safety-curriculum-guide2012.html

8. Wilson, Ross M., et al.Patient safety in developing countries: retrospective estimation of scale and nature of harm to patients in hospital. Bmj. 2012 Mar 13;344:e832

9. Maheshwari V. A study to assess Knowledge and attitude regarding hand hygiene amongst residents and nursing staff in a tertiary health care setting of Bhopal City. Journal of clinical and diagnostic research: JCDR. 2014 Aug;8(8): DC04-DC07.

10. Nair SS, Hanumantappa R, Hiremath SG, Siraj MA, Raghunath P. Knowledge, attitude, and practice of hand hygiene among medical and nursing students at a tertiary health care centre in Raichur,
India. ISRN preventive medicine" , "ISRN Prev Med. 2014;2014.

11. Kamble VS, Biradar SM, Takpere A, Reddy $\mathrm{S}$. Knowledge of hand hygiene practices among students of ESIC medical college, Gulbarga, Karnataka, India. Int J Community Med Public Health. 2016 Jan;3(1):95-98.

12. World Health Organization. World Alliance for Patient Safety. WHO guidelines on hand hygiene in health care , Geneva-27, WHO-Press 2009; WHO global survey of patient experiences in hand hygiene improvement251-257.

13. Bouwer $M$, Labuschagne $S$, Spamer $S$, Vermaak C, Zietsman LM, Steyn D, Joubert G. Knowledge of final-year medical students at the University of the Free State of hand hygiene as a basic infection control measure. South African Family Practice. 2018;60(3):74-78 